\title{
FISH COLLECTED DURING THE CORINDON II AND IV EXPEDITIONS
}

\author{
by \\ P. FOURMANOIR ${ }^{1}$ )
}

\section{INTRODUCTION}

The check-list of the fish collected during the CORINDON expeditions (COR. II: 20 sampling stations in the Makassar Strait; COR. IV: 7 stations in Piru and Ambon Bay in the Moluccas) ${ }^{2}$ ) is composed out of 110 species. Most of them are coming from depths between $250 \mathrm{~m}$ and $600 \mathrm{~m}$; a dozen of species were found at less deep stations; two Apodes, of which one is the very rare Promyllantor, were collected at a $1000 \mathrm{~m}$ depth.

The Ophidiiformes, the Pleuronectiformes and the Peristediidae are making a very interesting collection because of their great number of species.

Among the Percophidae, Bembrops filifera is reported for the first time outside its location in Hawaii; Acanthaphrites grandisquamis was known before only in the Arafura Stea and in the south of the China Sea. One will notice the big size of the Polyipnus spinifer and Astronesthes luicifer specimens.

The collection has been divided between the National Museum of Natural History of Paris and the Lembaga Oseanologi Nasional of Jakarta, excepting two or three species represented by a single specimen. So the only specimen of Branchiostegus is in Paris, while the rare Psychrolutes inermis is in Jakarta.

1) Marine biologist from GRSTOM, Laboratoire d'ichtyologie generale du Museum National d'Histoire Naturelle, 57, rue Cuvier. Paris, 75005.

$\left.{ }^{2}\right)$ The detailed check-list of the stations can be found in the Report on the Corindon Cruises by M.K. MOOSA. 


\section{HALOSAURIDAE}

Aldrovandia mediorostris (Gunther)

COR II; st 280: 1 sp. $430 \mathrm{~mm}$.

Inf. G.R. 18. Fins yellowish.

Aldrovandia rostrata (Gunther)

COR. II; st. 280: $1 \mathrm{sp}$. about $420 \mathrm{~mm}$ (the posterior part of the tail is broken).

Inf. G.R. 14. Fins dark.

\section{ALEPOCEPHALIDAE}

Alepocephalus (Lloydiella) bicolor Alcock

COR. IV; st. Ill 1: 2 sp. 154, $170 \mathrm{~mm}$; st. Ill 2: 1 sp. $210 \mathrm{~mm}$.

D. 21; A 28; 1. L. 63 (sp. $210 \mathrm{~mm}$ ).

Alepocephalus (Lloydiella) sp.

COR. II; st. 280: 1 sp. $270 \mathrm{~mm}$; st. 281: 1 sp. $221 \mathrm{~mm}$.

D. 22-24; A, 30-32; P. 10; 1. L. 86-90; inf. G.R. 11.

The species has similarities to Conocara macropterus (Vaillant 1888);

(D. 19; A. 34; P. 9; 1. L. 85).

\section{ARGENTINIDAE}

Nansenia sp.

COR. II; st. $276 ; 1$ sp. $180 \mathrm{~mm}$.

D. 10; A. 8; P. .15; inf. G.R. 17 - Height of body $24 \mathrm{~mm}$, head length 40. $\mathrm{mm}$.

Related to N. ardesiana Jordan \& Thompson, from Japan.

\section{CHLOROPHTALMIDAE}

Chlorophtalmus albatrossis Jordan \& Starks

COR. IV; st. IV 1: 1 sp. $145 \mathrm{~mm}$; st. V 2: $1 \mathrm{sp} .76 \mathrm{~mm}$.

1. L. 55-56; inf. G.R. 17.

Chlorophtalmus sp.

COR. IV; st. V 1: 1 sp. $69 \mathrm{~mm}$.

D. 10; A. 8; P. 18. 1. L. 45; inf. G.R. 16 - Snout length $4.1 \mathrm{~mm}$, diameter of orbit $5.5 \mathrm{~mm}$.

The tips of the dorsal and ventral fins are black. Superior caudal lobe with the first outer ray transparent, followed by two black rays. The two outer inferior caudal rays are only half-length black. 
FISH OF THE CORINDON II AND IV

Chlorophtalmus acutifrons Hiyama

COR. II; st. 271: 1 sp. 157 mm. - COR. IV; st. V 2: 2 sp. 120, 174 mm. 1. L. 52-53; inf. G.R. 14.

\section{ASTRONESTHIDAE}

Astronesthes lucifer Gilbert

COR. IV; st. I 2: $1 \mathrm{sp} .137 \mathrm{~mm}$.

D. 12; A. 19; P. 6; V. 7; G.R. 18 - Height of body 14; head length 20.5 ; orbit 6 ; upper jaw 14.5 ; interorbital width 7 ; barbel 12 ; snout length 4; tip of snout to dorsal origin 51 , to ventrals 44.5 origin of D. to base of caudal fin 48 .

Photophores: Op 2, Br 18; Is 8, PV 21 VAV 19, AC $2+7$, C4 (ventral row); OV 17, VAL 21 (lateral row).

Maxillary comblike teeth 24, mandibulary teeth 8 .

\section{MYCTOPHIDAE}

Diaplus suborbitalis Weber

COR IV; st. 1 2: 2 sp. 63, 74 mm (5).

The two specimens (typical S.E. Asia form) have 29 gillrakers $(10+1+18)$. Comments have been made (chiefly from Japan) of special abundance in continental waters and paucity far at sea (KAWAGUCHI \& SHIMIZU 1978). Adult females have not been found near the surface.

Diaphus garmani Gilbert

COR. IV; st. IV 1: 4 ㅇ 56, 59, 61, 63 mm; 2 ^ 55, 56 mm.

\section{NEOSCOPELIDAE}

Neoscopelus porosus Arai

COR. IV; st. I 1: 2 sp. 120, $132 \mathrm{~mm}$; st. Ill 1: 3 sp. 73, 89, $104 \mathrm{~mm}$; st. Ill 2: 2 sp. $120,130 \mathrm{~mm}$.

Neoscopelus microchir Matsubara

COR. II; st. 274: 3 sp. 70, 75, $120 \mathrm{~mm}$.

\section{STERNOPTYCHIDAE}

Polyipnus spinifer Borodinula

COR. IV; st. IV 1: 4 sp. 51, 54, 66, 69 mm. 


\section{CEPOLIDAE}

Sphenanthias pectinifer Myers

COR. II; st. 273: 1 sp. $102 \mathrm{~mm}$, st. 271: 1 sp. $98 \mathrm{~mm}$.

D. 27 ; 1. L. $28 ; 32$ ser. of scales; G.R. $18+27$.

Sphenanthias sp.

COR. II; st. 271: 1 sp. $47 \mathrm{~mm}$.

D. 25 (III, 22); A. I, 15; P. 21; 1. L. about 33; tot. G.R, 40.

The dorsal fin has a black margin between the first and the sixth ray. The species differs from $S$. sibogae in the coloration of the dorsal and more scales.

\section{CHAMPSODONTIDAE}

Champsodon vorax Gunther

COR. II; st. 267: 10 sp. 73-90 mm.

\section{SYNODONTIDAE}

Harpadon microchir Gunther

COR. IV; st. 11:1 sp. $275 \mathrm{~mm}$; st. I 2: 2 sp. 250, $280 \mathrm{~mm}$.

Saurida longimanus Norman

COR. II; st. 295: $1 \mathrm{sp} .114 \mathrm{~mm}$.

Saurida tumbil (Bloch)

COR. II; st. 201: 1 sp. $430 \mathrm{~mm}$.

\section{PEGASIDAE}

Pegasus papilio Gilbert

COR. II; st. 292: 1 sp. $42 \mathrm{~mm}$.

ZEIDAE

Cyttopsis cypho (Fowler) COR.

II; st. 271: 4 sp. $70 \mathrm{~mm}$.

\section{DIRETMIDAE}

Diretmus argenteus Johnson

COR. II; st. 276: 1 sp. $90 \mathrm{~mm}$. 


\section{FISH OF THE CORINDON II AND IV}

\section{TRACHICHTHYIDAE}

Paratrachichthys latus Fowler

COR. II; st. 267: 3 sp. 35, 38, $39 \mathrm{~mm}$.

D. Ill, 15; A. II, 9; P. 12.

Hoplostethiis sp.

COR. II; st. 271: 1 sp. $90 \mathrm{~mm}$.

D. VI, 13; A. Ill, 10; P. 14; G.R. 20.

The average G.R. number is 24 in $H$. mediterraneus, the Makassar specimen has only 20 gill rakers.

\section{POLYMIXIIDAE}

Polymixia berndti Gilbert

COR. II; st. 271; 1 sp. $103 \mathrm{~mm}$.

D. V, 30; A. IV, 15; 1. L. 33; scales ser. 60; G.R. $4+1+7$.

\section{CHEILODIPTERIDAE}

Bathysphyraenops declivifrons Fedoryako

COR, IV; st. I 1: 1 sp. $54 \mathrm{~mm}$; st. I 2: 2 sp. 54 mm, 2 sp. $58,59 \mathrm{~mm}$.

\section{APOGONIDAE}

Apogon catalai Fourmanoir

COR. II; st. 292: 1 sp. $42 \mathrm{~mm}$.

Apogon quadrifasciatus Cuvier

COR. II; st. 203: 2 sp. $64 \mathrm{~mm}$.

Jaydia smithi Kotthaus

COR. II; st. 295: 1 sp. $50 \mathrm{~mm}$.

Jaydia queketti (Gilchrist)

COR. II; st. 203: 3 sp. 72, 82, 100 mm.

Synagrops japonicus Steindacmier

COR. II; st. 271: 2 sp. 88, 92 mm - COR. IV; st. V 1: 3 sp. 60, 63, 67 mm;

st. V 2: 1 sp. $100 \mathrm{~mm}$.

Synagrops philippinensis (Gunther)

COR. II, st. 273: $1 \mathrm{sp} .66 \mathrm{~mm}$ - COR. IV; st, V 1: 2 sp. 61, $66 \mathrm{~mm}$.

\section{SERRANIDAE}

Chelidoperca hirundinacea (Valenciennes)

COR. II; st. 273: 1 sp. $63 \mathrm{~mm}$. 
Pseudanthias (elongtus Franz?)

COR. II; st. 206: 1 sp. $79 \mathrm{~mm}$.

Plectranthias japonicus Steindachner

COR. II; st. 273: 1 sp. $62 \mathrm{~mm}$ - COR. IV; st. V 2: 1 sp. $59 \mathrm{~mm}$.

\section{NEMIPTERIDAE}

Scolopis tosensis Kamohara COR.

II; st. 273: 1 sp. $52 \mathrm{~mm}$.

\section{LUTJANIDAE}

Tropidinius argyrogrcummicus Valenciennes

COR. II; st. 271: 2 sp. 82, 175 mm; st. 273: 3 sp. 125, 143, $163 \mathrm{~mm}$.

\section{EMMELICHTHYIDAE}

Emmelichthys nitidus nitidus Richardson

COR. II; st. 273: 2 sp. 112, $113 \mathrm{~mm}$

D. XI, I 10; A. Ill, 9; 1. L. 89; G.R. $10+1+25$.

\section{BRANCHIOSTEGIDAE}

Branchiostegus wardi Whitley

COR. II; st. 273: 1 sp. 164 mm.

\section{NOMEIDAE}

Cubiceps squamiceps (Lloyd)

COR. II; st. 246: 1 sp. $136 \mathrm{~mm}$.

Ariomma indica (Day) COR. II; st.

240: 1 sp. $173 \mathrm{~mm}$.

\section{GEMPYLIDAE}

Neoepinnula orientalis (Gilchrist \& Bonde)

COR. II; st. 274: 1 sp. $153 \mathrm{~mm}$.

\section{OPHIDIIDAE (NEOBYTHITINAE)}

Glyptophidium argenteum Alcock

COR. II; st. 240: 10 sp. 210-250 mm.

Glyptophidium sp.

COR. II; st. 240: $12 \mathrm{sp.}$

D. 142-148; A. 114-122; P. 22-23; inf. G.R. 16-17, tot. G.R. 24-25.

Neobythites steatiticus Alcock

COR. II; st. 273: 1 sp. $132 \mathrm{~mm}$. 
Homostolus acer Smith \& Radcliffe COR. IV; st. I $1: 2$ sp. $155,160 \mathrm{~mm}$; st. $12: 1$ sp. $147 \mathrm{~mm}$.

Monomitopus garmani (Smith \& Radcliffe)

COR. II; st. 200: $1 \mathrm{sp} .197 \mathrm{~mm}$.

D. 97; P. 32; C. 8; tot, G.R. 22 (inf. 16).

Monomitopus sp.

COR. II; st. 240: 1 sp. $135 \mathrm{~mm}$.

Neobythites longipes Smith \& Radcliffe

COR. IV; st. I 2: 2 sp. 191, 192 mm.

Neobytjiites fasciatus Smith \& Radclliffe

COR. II; st. 246: 2 sp. 25, $164 \mathrm{~mm}$ - COR. IV; st. I 2: 1 sp. $147 \mathrm{~mm}$;

st. Ill 1: 1 sp. $150 \mathrm{~mm}$.

Lamprogrammus macropterus Smith \& Radcliffe

COR. II; st. 231: 1 sp. 460 mm (Head 100, Depth 100)

D. about 115; P. 21; C. 8; G.R. 16.

Dicrolene tristis Smith \& Radcliffe

COR, II; st. 200: 2 sp. 125, 162 mm.

\section{BYTHITIDAE (BYTHITINAE)}

Dicplacanthopoma brunnea Smith \& Radcliffe

COR. IV; st. IV 1: $1 \mathrm{sp} .168 \mathrm{~mm}$.

\section{CARAPIDAE (PYRAMODONTINAE)}

Pyvamodon ventraMs (Smith \& Radcliffe) COR, II; st. 267: 1 sp. $120 \mathrm{~mm}$.

\section{PLATYCEPHALIDAE}

Elates ransonetti (Steindachner)

COR. II; st. 203: 1 sp. $95 \mathrm{~mm}$.

Platycephalus sculptus (Gtinther)

COR. II; st. 206: 1 sp. 115, 120 mm.

\section{HOPLICHTHYIDAE}

Hoplichthys prosemwn (Fowler)

COR. IV; st. V 2: 2 sp. 140, 149 mm. 


\section{MUGILOIDIDAE}

Parapercis alboguttata (Gunther)

COR. II; st. 267: 1 sp. $128 \mathrm{~mm}$.

D. V, 22; A. 19; P. 18; 1. L. 63; G.R. 8 rud. +13.

Palatine teeth absent, IVth dorsal spine the longest.

\section{PERCOPHIDAE}

Bembrops eaaiMmucula Steindachner

COR. II; st. 267: 3 sp. 105, 125, 135 mm.

Bemhrops filifera Gilbert

COR. II; st. 271: 1 sp. $165 \mathrm{~mm}$.

D. VI, 15; A. 17; P. 27; 1. L. 65-66.

Chrionema chryseres Gilbert

COR. IV; st. V 2: 1 sp. $172 \mathrm{~mm}$.

Acanthaphrites grandisquamis Gunther (Fig. 1-3)

COR. IV; st.-V 2: 3 sp. 79, 101, $124 \mathrm{~mm}$.

D. V. 22-23: A. 28-29: P. 20-21; V. 6; 1. L. 34-36; depth 8. 2.

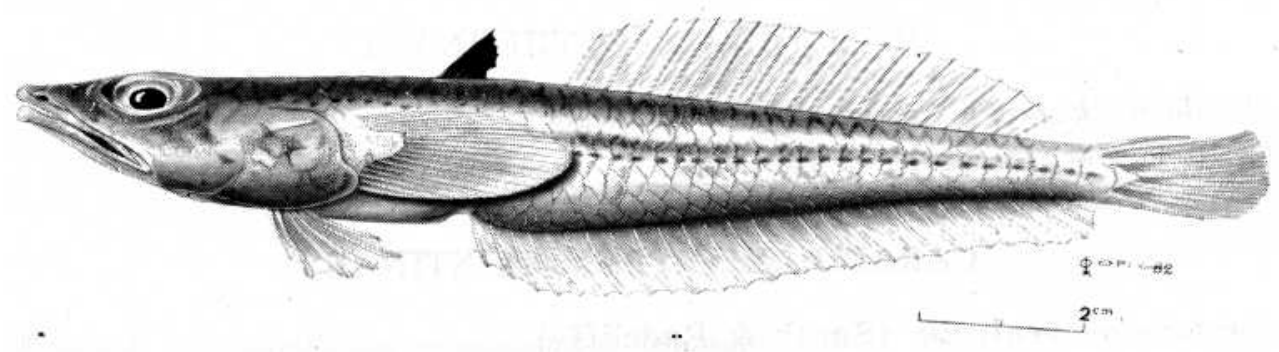

Figure 1. Acanthaphrites grandisquamis Gunther. Lateral view. L.S. $124 \mathrm{~mm}$.

Body moderately elongated. Snout short, notched and spinous. Maxillary reaching middle of eye. Opercular flap well developed, covering the origin of inferior pectoral rays. Upper margin with a strong spine. Maxillary teeth, 3-4 rows of about 56 villiform teeth. Each lateral portion of vomer with a small patch of 5-8 teeth.

Last dorsal ray small, last anal ray minute at a short distance of origin of caudal. Scales of lateral line with four or five deep indentations. The other scales, a little ctenoid, more or less square. Nine scales before dorsal origin. Cheeks scaly.

Coloration in formaline: First dorsal black, about a dozen of irregular, grey-olive cross bands, not extending beyond lateral line. 


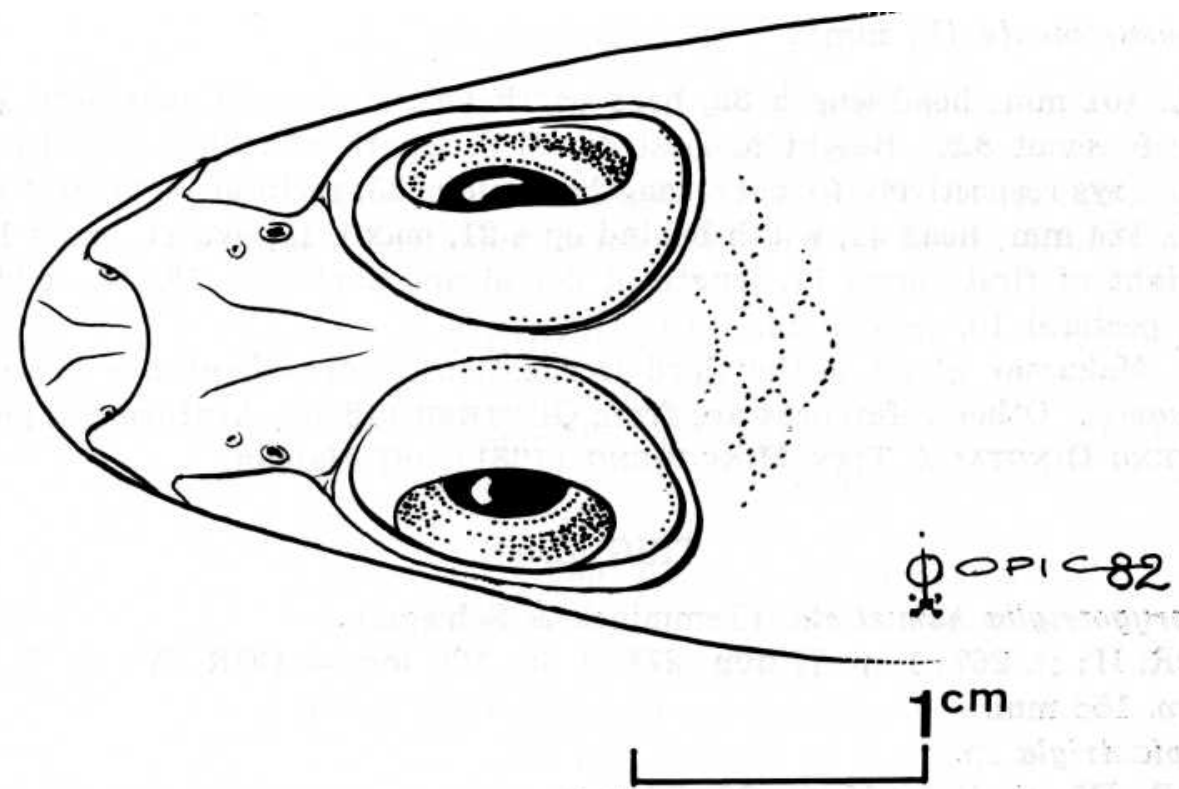

Figure 2. Acanthaphrites grandisquamis Günther. Head, dorsal view.

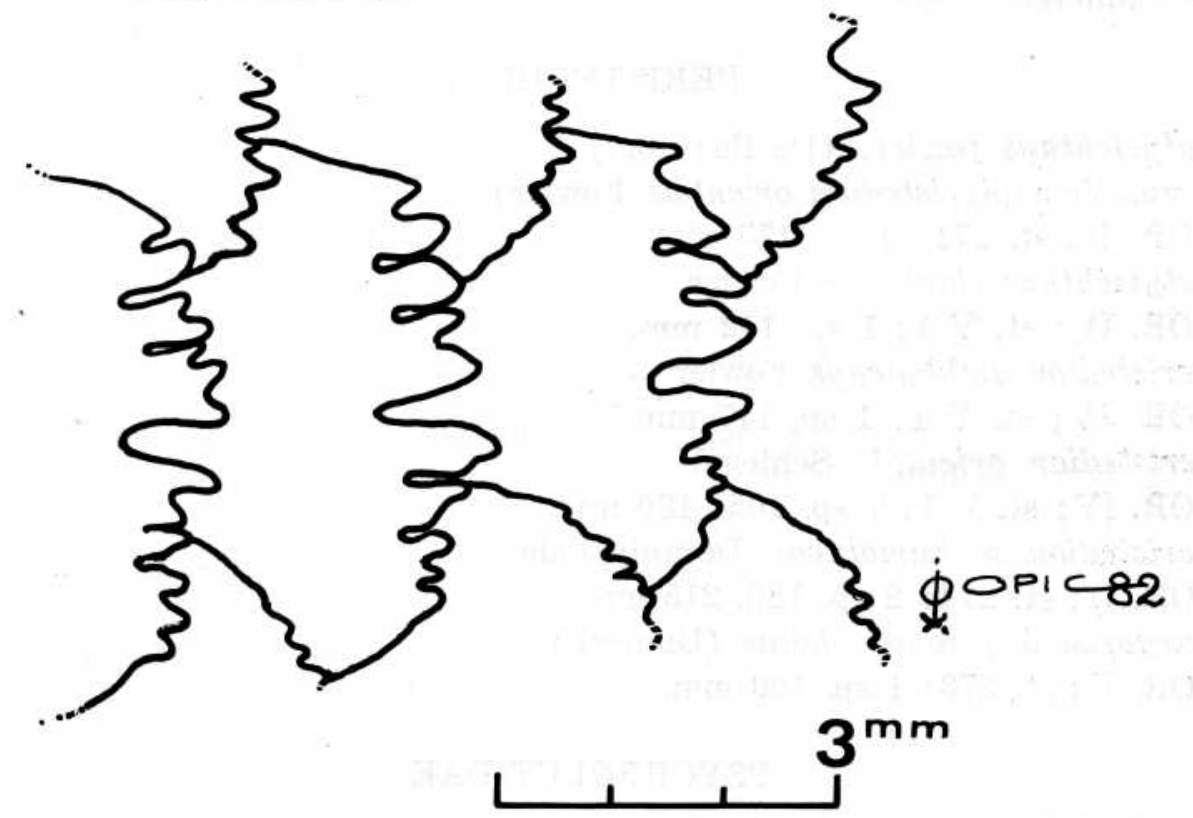

Figure 3. Aeanthaphrites grandisquamis Günther. 12th, 13th and 14 th lateral line scales, left side. 
Measurements (in $\mathrm{mm}$ ):

S.L. $101 \mathrm{~mm}$; head length 32 , head width behind eyes 15 , maxillary 13 , eye 9, snout 8.5. Height of first dorsal 9, length of middle dorsal and anal rays respectively 10 and 8, length of caudal 15, pectoral 14, ventral 10 . S.L. $124 \mathrm{~mm}$; head 41, width behind eyes 21, maxil. 18, eye 11, snout 11 . Height of first dorsal 11, length of dorsal and anal rays 13, 10, caudal 20, pectoral 19, ventral 14 .

Makassar Strait is the third localization of Acanthaphrites grandisquamis. Other references are from GuNTHER (1880), Arafura Sea and Cheng Qingtai \& Tian MingCheng (1981), off Hainan.

\section{TRIGLIDAE}

Pterygotriglia hemisticta (Temminck \& Schlegel)

COR. II; st. 267: 1 sp. 77 mm; 271: 1 sp. $120 \mathrm{~mm}$ - COR. IV; st. V 2: $1 \mathrm{sp} .155 \mathrm{~mm}$.

Lepidotrigla sp.

COR. IV; st. V 1: 10 sp. $68-73 \mathrm{~mm}$.

D. VIII, 11; A. 12; 1. L. 52 - 53; rostral spines a little diverging ${ }^{1}$, as long as diameter of eye.

\section{PERISTEDIIDAE}

Satyrichthys fowleri (De Beaufort) (syn. Nemapteristedion orientate Fowler)

COR. II; st, 271: $1 \mathrm{sp} .175 \mathrm{~mm}$.

Satyrichthys clavilapis Fowler

COR. IV; st. V 1: 1 sp. $142 \mathrm{~mm}$.

PeHstedion amblygenys Fowler

COR. IV; st. V 1: 1 sp. $147 \mathrm{~mm}$.

Peristedion orientals Schlegel

COR. IV; st. V 1: 7 sp. 105-130 mm.

PeHstedion pothumaluvae Deraniyagala

COR. II; st. 214: 2 sp. 180, 215 mm.

Gargariscus pHonocephalus (Dumeril)

COR. II; st. 273: 1 sp. $100 \mathrm{~mm}$.

\section{PSYCHROLUTIDAE}

Psychrolutes inermis (Vaillant)

COR. II, st. 214: 1 sp. (L.O.N. Ichthyological Laboratory, Jakarta). 


\section{FISH OF THE CORINDON II AND IV}

\section{CEPHALACANTHIDAE}

Cephalacanthus orientalis (Cuvier)

COR. II; st. 203: 1 sp. $52 \mathrm{~mm}$.

\section{SCORPAENIDAE}

Pterois volitans Linne

COR. II; st. 295: 1 sp. $85 \mathrm{~mm}$.

Dendrochirus bellus (Jordan \& Hubbs)

COR. II; st. 203: 1 sp. $57 \mathrm{~mm}$; st. 295: 1 sp. $90 \mathrm{~mm}$.

Neocentropogon aeglefinus (Weber)

COR. II; st. 273: 3 sp. 64, 66, 73 mm.

Minous coccineus Alcock

COR. II; st. 295: 2 sp. 53, 79 mm.

Scorpaena sp.

COR. II; st. 295: 3 sp. 43, 71, 73 mm.

Setarches longimanus Alcock

COR. II; st. 267: 1 sp. 92 mm.

Setarches guentheri Johnson

COR. II; st. 269: 1 sp. $60 \mathrm{~mm}$; st. 27: 2 sp. $59 \mathrm{~mm}$; st. 273: 1 sp. $62 \mathrm{~mm}$;

COR. IV; st. V 1: $35 \mathrm{sp} .36-52 \mathrm{~mm}$.

Lioscorpius longiceps Gunther

COR. IV; st. V 2: 2 sp. 36, 38 mm.

Erisphex potti (Steindachner)

COR. IV; st. V 1: 1 sp. $30 \mathrm{~mm}$.

\section{URANOSCOPIBAE}

Uranoscopus japonicus Houltuyn

COR. II; st. 267: 1 sp. 90 mm; st. 271: 1 sp. $200 \mathrm{~mm}$.

Uranoscopus oligialepis Bleeker

COR. II; st. 295: 1 sp. $70 \mathrm{~mm}$.

\section{CALLIONYMIDAE}

Synchiropus altivelis (Temminck \& Schlegel)

COR. II; st. 271: 3 sp. 75, 90, 95 mm.

Callionymus octostigmatus Fricke

COR. II; st. 205: 3 sp. 25, 27, 43 mm. 


\section{BOTHIDAE}

Chascanopsetta lugubris Alcock

COR. IV; st. V 1: 1 sp. $200 \mathrm{~mm}$; st. V 2: 1 sp. $148 \mathrm{~mm}$.

Laeops gracilis Fowler

COR. IV; st. V 1: 2 sp. $97,115 \mathrm{~mm}$.

D. 109; A. 93; P. 14; 1. L. 98 (sp. $97 \mathrm{~mm})$.

D. 104; A. 91; 1. L. 95 (sp. 115 mm).

Arnoglossus sp.

COR. II; st. 271: 2 sp. 150, $164 \mathrm{~mm}$.

D. 110 ; A. 86 ; 1. L. 77 ; P. 21 (sp. 164 mm).

D. 107 ; A. 83 ; 1. L. 80 ; P. 15 (sp. $150 \mathrm{~mm}$ ).

Arnoglossus elongatus Weber

COR. II; st. 271: 2 sp. 123, $125 \mathrm{~mm}$.

D. 102; P. 12; 1. L. 65.

\section{PLEURONECTIDAE}

Poecilopsetta plinthus (Jordan \& Starks)

COR. II; st. 271: 1. sp. $93 \mathrm{~mm}$.

Poecilopsetta praelonga Alcock

COR. II; st. 271: $1 \mathrm{sp} .90 \mathrm{~mm}$.

Samariscus huysmani Weber

COR. II; st. 215: 2 sp. 75, $92 \mathrm{~mm}$.

Brachypleura novaezeekmdiae Giinther

COR. II; at. 203: 3 sp. $82,85,86 \mathrm{~mm}$.

\section{SOLEIDAE}

Aseraggodes filiger Weber

COR. II; st. 203: 3 sp. $65 \mathrm{~mm}$.

Achirus hartzfeldi Bleeker

COR. II; st. 258: 1 sp. $85 \mathrm{~mm}$.

Heteromycteris hartzfeldi (Bleeker)

COR. II; st. 258: 1 sp. $85 \mathrm{~mm}$.

Heteromycteris japonicus (Temminck \& Schlegel)

COR. II; st. 258: 1 sp. $83 \mathrm{~mm}$.

Zebrias zebra (Bloch)

COR. II; st. 203: 1 sp. $90 \mathrm{~mm}$; st. 295: 1 sp. $235 \mathrm{~mm}$. 


\section{FISH OF THE CORINDON II AND IV}

\section{CYNOGLOSSIDAE}

Symphurus sp.

COR. II; st. 269: 1 sp. $103 \mathrm{~mm}$.

D. 87; Gaud. 13; 1. L. 90.

Apparently near S. gilesi.

Cynoglossus sp.

COR. II; st. 271: 1 sp. $122 \mathrm{~mm}$.

D. 97; Caud. 10; middle 1. L. 76, 11 scales between the two 1. L.

Apparently near C. cynoglossus, but it is a little more elongated and dorsal and anal fins are much darker.

\section{ONCHOCEPHALIDAE}

Halieuta, stellata (Vahl.)

COR. II; st. 267: 1 sp. $90 \mathrm{~mm}$; st. 273: $1 \mathrm{sp} .85 \mathrm{~mm}$.

Dibranchius infranudus De Beaufort \& Briggs

COR. II; st. 276: 1 sp. $52 \mathrm{~mm}$.

\section{CHAUNACIDAE}

Choimmx fimbriatus Hilgendorf

COR. IV; st. I 2: 1 sp. $88 \mathrm{~mm}$.

\section{SYNAPHOBRANCHIDAE}

Ilyophis brunneus Gilbert

COR. II; st. 231: 1 sp. $475 \mathrm{~mm}$.

Total 1. L. pores 130, preanal 1. L. pores 34.

CONGRIDAE

Promyllantor pwpureus Alcock

COR. II; st. 281: $1 \mathrm{sp} .450 \mathrm{~mm}$.

Bathymyrus japonicus

COR. II; st. 271: 2 sp. $300 \mathrm{~mm}$.

Congrina sp.

COR. II; st. 271: 1 sp. $350 \mathrm{~mm}$; st. 273: 1 sp. $380 \mathrm{~mm}$.

\section{SIMENCHELYDAE}

Symenchelis parasiticus Gill

COR. II; st. 280: 1 sp. $165 \mathrm{~mm}$ 


\section{P. FOURMANOIR}

\section{MACROURIDAE}

Coelorhynchus argus Weber

COR. IV; st. V 1: 1 sp. $118 \mathrm{~mm}$.

Hymenocepalus striatissimtis Smith \& Radcliffe

COR. IV; st. V 2: 3 sp. 110, 115, $125 \mathrm{~mm}$.

Mataeocephalus nigrescens Smith \& Radcliffe

COR. II; st. 240: $1 \mathrm{sp}, 170 \mathrm{~mm}$.

Coryphaenoides microps (Smith \& Radcliffe)

COR. IV; st. I 1: 5 sp. $170-190$ mm; st. III 1: 3 sp.140, 150, 182

$\mathrm{mm}$;

st. III 2: $3 \mathrm{sp} .210 \mathrm{~mm}$.

Gadomus introniger Gilbert \& Hubbs

COR. II; st. 280: 2 sp. 300, $390 \mathrm{~mm}$.

\section{REFERENCES}

AmAOKA, K. and al. 1982. Fishes of the Kyushu-Palau ridge and Tosa Bay. Japan fisheries resource conservation association. Tokyo, $435 \mathrm{p} ., 222$ figs.

Alcock, M.B. 1889. A descriptive catalogue of the Indian deep-sea fishes in the Indian Museum collected by the Royal Indian marine survey ship Investigator. Calcutta, 220 pp. 1 map.

De Beaufort, L.F. and W.M. Chapman 1951. Percomorphi (concluded) Blennoidea IX. In: The fishes of the Indo-Australian Archipelago, 484 p., 89 figs.

Cantwell, G.E. 1064. Revision of the Genus Parapercis. Pacif. Sci. 18 (3): 2S9--2\&0, 9 figs.

COHEN, D.M. and J.G. NIELSEN 197\&. Guide to the identification of genera of the ehiostegidae and Malacanthidae), with descriptions of two species. NO A A Techn. Rep. NMFS Circ. 417, 7:2 p., 103 figs.

CoHEn, DJM. 1958. Nansenia Candida, a new species of Argentinid fish from the North Pacific, with notes on other species of Nansenia. Stanford ichthyol. Bull. 7 (3): 52-.571, 1 fig.

DoOLEY, J.K. 1078. Systematics and biology of the Tilefishes (Perciformes: Branchiostegidae and Malacanthidae), with descriptions of two new species. NO A A Techn. Rep. NMFS Circ. 411, 78 p., 44 figs.

GUNTHER, A. 1880. Report on the shore fishes procured during the voyage of H.M.S. Challenger in the years 1873-76. In: Report on the scientific results of the voyage of H.M.S. Challenger during the years 1878-76, Zoology I (6): 1 - 82; pis. 1 - 32.

Gilbert, C.H. 1903. The deep-sea fishes of the Hawaiian islands. U.S. Fish. Com. Bull, 23: 578 - 713.

HeEmSTRA, P.C. 1980. A revision of the Zeid fishes (Zeiformes: Zeidae) of South Africa. Ichtchyol. Bull. Rhodes Univ. 41, 16 p., 2 pls., 5 figs. 


\section{FISH OF THE CORINDON II AND IV}

HeEmStRA, P.C, and J.E. RANDAlL 1977. A revision of the Emmelichthyidae (PiseesPerciformes). Aust. J. Mar. Freshw. Res., 28 (3): 361'-396.

KAWAguchi, K. and H. ShimizU 1978. Taxonomy and distribution of the Lanternfishes genus Diaphus (Pisces, Myctophidae) in the Western Pacific, East Indian Ocean and the Southeast Asian Seas. Bull. Ocean. Res. Inst. Univ. Tokyo, (10): 1-145.

MENON, A.G.K. 1977. A systematic monograph of the tongue soles of the genus Cynoglossus Hamilton. Smithson. Contrib. Zool., 23i8: 1-11219, 21 pi.

NAFPAKTITIS, B.J. 1978. Systematies and distribution of lanternfishes of the genera Lobianchia and Diaphus (Myctophidae) in the Indian Ocean. Nat. Hist. Mus. Los Angeles County Sci. Bull. 30, 92! p., 82 figs.

Nelson, J.S. 1982. Pteropsaron heemstrae and Osopsaron natalensis (Perciformes, Percophidae), new fish species from South Africa with comments on Squamicreedia obtusa from Australia and on classification of the Subfamily Hemerocoetinae. J.L.B. SMITH Inst. Ichthyol, sp. Publ. 25, 11 pp., 7 figs.

Nelson, J.S. 1982. Two new South Pacific fishes of the genus Ebinania and contributions to the systematics of Psychrolutidae (Scorpaeniformes). Can. J. ZooL. 60 (6): 1470-1504.

NORMAN, J.R. 1I934. A systematic monograph of the Flatfishes \{Het\&rosomata). British Mus. Ed., 437 pp., 317 figs.

PARR, A.E. 1952, Revision of the species currently referred to Alepocephalus, Halisauriceps, Bathythroctes and Bajacalifornia with introduction of two genera. Bull Mus. com. Zool. Harv., 107) (4): 255 - 269.

Qingtai, C. and T. MingCheng 1981. A preliminary report of the deep-sea fishes of the South China Sea. Academia Sinica, Contribution 527. Studia marina sinica, 18: 233-275, 32 figs.

Susuki, K. and S. KIMURA 1980. First record of the deep-sea cottid fish Psychrolutes inermis from Japan. Jpn. J. Ichthyol., 27 (1): 77-81.

VAillant, L. 1®88. Poissons. In: Elxpeditions scientifiques du Travailleur et du Talisman pendant les annees 1880-83. Paris: 406 pp., 28 pls. 